\title{
Producción de conidios y clamidosporas de los hongos Duddingtonia flagrans y Monacrosporium thaumasium en diferentes medios sólidos
}

\author{
Produção de conídios e clamidósporos de Dunddingtonia flagrans e \\ Monacrosporium thaumasium em diferentes meios sólidos
}

\author{
Manoel Eduardo da Silva ${ }^{1,2}$, Fábio Ribeiro Braga ${ }^{1,3}$, Luana Alcântara Borges', \\ Paulo de Oliveira ${ }^{4}$, Walter dos Santos Lima ${ }^{5}$, Jackson Victor de Araújo ${ }^{1 *}$

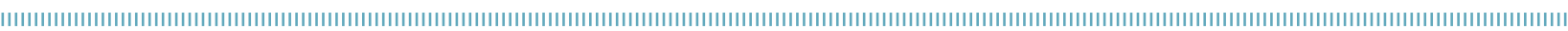

RESUMEN: Las especies Duddingtonia flagrans y Monacrosporium thaumasium son micro-hongos considerados promisorios agentes del control biológico de parásitos. Bajo condiciones adversas como la falta de nutrientes, estos hongos producen esporas capaces de sobrevivieren después de pasar por el tracto gastrointestinal de los animales. La formación de estas estructuras es una característica deseable ya que promueve la sobrevivencia y la diseminación de los hongos para propósitos de biocontrol. El objetivo de este estudio fue evaluar la producción de esporas de dos especies de hongos nematófagos D. flagrans (aislados AC001 y CG722) y M. thaumasium (NF34A). Estos fueron cultivados en los subproductos agroindustriales, que tenían el intento de identificar el mejor medio para uso en programas de biocontrol de nematodos. Diferentes volúmenes $(10,15$ y $20 \mathrm{~mL})$ de masa micelial fueron utilizados como inóculos iniciales y adicionados a 100 gramos de medios de crecimientos sólidos (sémola de arroz - QA; sémola de maíz - QM; bagazo de caña - BC; paja de arroz - PA y cascara de café - CC) y mantenidos a $25^{\circ} \mathrm{C}$ en la obscuridad para evaluar la producción de esporas. Los aislados AC001 y CG722 mostraron las mejores producciones en el medio de la QA $(\mathrm{p}<0,05)$. El volumen de $20 \mathrm{~mL}$ de masa micelial utilizado como inóculo inicial proporcionó una mayor recuperación de esporas. El aislado NF34A presentó una baja o nula producción de estructuras reproductivas en los diferentes volúmenes y medios de crecimientos utilizados. La mejor producción de esporas se obtuvo utilizando subproductos de la agroindustria con mayor densidad proteica y energética.

PALABRAS CLAVE: esporas; hongos nematófagos; control biológico; subproductos agroindustriales.
RESUMO: As espécies Duddingtonia flagrans e Monacrosporium thaumasium são microfungos considerados promissores agentes de controle biológico de parasitos. Sob condiçóes adversas, como a falta de nutrientes, estes fungos produzem esporos capazes de sobreviverem após a passagem pelo trato gastrintestinal dos animais. A formação destas estruturas é uma característica desejável, já que promove a sobrevivência fúngica e a sua disseminação para fins de biocontrole. Este estudo teve como objetivo avaliar a produção de esporos das espécies de fungos nematófagos D. flagrans (isolados AC001 e CG722) e M. thaumasium (NF34A). Estes foram cultivados em subprodutos agroindustriais, a fim de identificar o melhor meio para uso em programas de controle biológico de nematoides. Diferentes volumes de massa micelial (10, 15 e $20 \mathrm{~mL})$ foram utilizados como inóculo inicial e adicionados sobre 100 gramas dos meios de crescimento sólidos (quirera de arroz - QA; quirera de milho - QM; bagaço de cana - BC; palha de arroz - PA e casca de café - CC) e mantidos a $25^{\circ} \mathrm{C}$ ao abrigo da luz para avaliar a produçáo de esporos. Os isolados AC001 e CG722 mostraram as melhores produçóes no meio QA ( $\mathrm{p}<0,05)$. O volume de $20 \mathrm{~mL}$ de massa micelial usado como inóculo inicial permitiu maior recuperação de esporos. O isolado NF34A apresentou produção baixa ou nula de estruturas reprodutivas nos diferentes volumes e meios de crescimento utilizados. A melhor produção de esporos foi obtida nos subprodutos agroindustriais com maior densidade proteica/energética.

PALAVRAS-CHAVE: esporos; fungos nematófagos; controle biológico; subprodutos agroindustriais.

'Departamento de Veterinária; Universidade Federal de Viçosa (UFV) - Viçosa (MG), Brasil.

${ }^{2}$ Instituto Técnico de Agropecuária e Cooperativismo; Empresa de Pesquisa Agropecuária de Minas Gerais (ITAC/EPAMIG) - Pitangui (MG), Brasil.

${ }^{3}$ Universidade Vila Velha (UVV) - Vila Velha (ES), Brasil.

${ }^{4}$ Sede da EPAMIG - Belo Horizonte (MG), Brasil.

${ }^{5}$ Instituto de Ciências Biológicas; Universidade Federal de Minas Geais (UFMG) - Belo Horizonte (MG), Brasil.

*Autor correspondente: manoelsilva@epamig.br

Recibido en: 04/10/2013. Acepto en: 16/10/2015 


\section{INTRODUCCIÓN}

Los hongos nematófagos producen esporas o conidios de resistencias conocidas como clamidosporas. Estas últimas, una vez que son administradas oralmente a los animales, poseen la capacidad para resistir la degradación gastrointestinal de los rumiantes y otros herbívoros (TAVela et al., 2011) y son eliminadas en las heces, manteniendo la suya capacidad germinativa y de colonización. Ellas ejercen una actividad depredadora y destruyen a los estadios parasitarios de nematodos (Silva et al., 2013) en las heces. Este sistema permite interrumpir el ciclo biológico de los parásitos (Araújo et al., 2004). Según Scholler; Rubner (1994), la formación de clamidosporas es una respuesta a condiciones de crecimiento desfavorables, como la deficiencia de nutrientes, y aunque los hongos no requieren de grandes cantidades de nutrientes para sobrevivieren, conocer que medios son favorables para una mayor capacidad de esporulación es una necesidad para su uso en los sistemas de control de nematodos parásitos del ganado (LARSEN, 2002; Araújo et al., 2004).

La esporulación fúngica abundante es un factor importante para la diseminación y sobrevivencia, además de ser deseable en programas de control biológico (ARAújo et al., 2001; Waller et al., 2001). Para el suyo crecimiento, los hongos requieren un conjunto de condiciones ambientales; sin embargo, la concentración de nutrientes en los medios de cultivo es lo que determinan la calidad y la cantidad del envejecimiento del hongo y si predominara la esporulación, favorecida por el agotamiento nutricional o crecimiento micelial (Dhingra; Sinclair, 1995). Según Larsen (1991), la temperatura óptima al desarrollo de Duddingtonia flagrans es del $30^{\circ}$ con una gran producción de esporas, pero en inferiores a $25^{\circ} \mathrm{C}$, existe crecimiento fúngico.

La especie $D$. flagrans tiene un elevado potencial para la producción de clamidosporas intercaladas a sus hifas y sus numerosos conidios formados en las extremidades de los conidióforos que son estructuras reproducibles (SANYAL et al., 2008). Los conidios son caracterizados a través de su morfología, la cual puede variar de elípticos a ovoides, aunque las clamidosporas son altamente resistentes y formadas en condiciones adversas de crecimiento y las hifas forman redes adhesivas relativamente cortas que atacan y destruyen rápidamente las larvas de los nematodos (BRAGA, 2008; CRuz et al., 2011).

El Monacrosporium thaumasium produce solamente un conidio en la extremidad apical de cada conidióforo, lo cual es generalmente erecto, transparente y fusiforme, con dos o más septos transversales y una célula intermediaria que es más grande en las extremidades (Liv; ZHANG, 1994; ZHANG et al., 1996, Araújo et al., 2004). Las especies del género Monacrosporium poseen hifas vegetativas septadas y ramificadas que las utilizan para depredar nematodos, por medio de redes adhesivas, nódulos y redes tridimensionales adhesivas o anillos constrictores (SaXena; MitTal, 1995).

Este trabajo tuvo como objetivo evaluar la producción de esporas/clamidosporas de hongos depredadores de las especies D. flagrans (aislados CG722 y AC001) y M. thaumasium (NF34A) en diferentes sustratos sólidos, como la sémola de arroz (QA); sémola de maíz (QM); el bagazo de caña con melaza de caña (BC); paja de arroz con melaza de cańa (PA) y cáscara de café con melaza de cańa (CC), considerados como subproductos de la agroindustria. Esto fue hecho para la determinación del mejor medio para la producción de esporas a su aplicación en programas de control biológico de las helmintiasis de bovinos en pruebas de pastoreo en campo.

\section{MATERIALES Y MÉTODOS}

\section{Organismos fúngicos}

Se utilizaron dos aislados fúngicos de suelos brasileños de la especie D. flagrans (AC001 y CG722) y uno de la M. thaumasium (NF34A), originarios de la micoteca del Laboratorio de Parasitología del Departamento de Veterinaria en la Universidad Federal de Viçosa, en Brasil, y mantenidos a $4^{\circ} \mathrm{C}$ en la obscuridad en tubos de ensayo, conteniendo medio de harina de maíz agar al 2\% (HMA 2\%).

\section{Producción de estructuras fúngicas}

Para evaluar la producción de conidios y clamidosporas, se utilizaron diferentes volúmenes de masa micelial $(10,15 \mathrm{y}$ $20 \mathrm{~mL}$ ). El criterio para establecer los inóculos iniciales al crecimiento de los aislados fúngicos AC001, CG722 y NF34A se basó en una prueba previa con diferentes medios líquidos, $\mathrm{pH}$ y tiempos de cultivo. Los volúmenes mencionados fueron aplicados como inóculo inicial, a los que se adicionaron 100 gramos de medios sólidos de crecimiento (QA; QM; BC; PA y CC), que fueron esterilizados a $120^{\circ} \mathrm{C}$ y 1,5 atm por 15 minutos y acondicionados en matraces Erlenmeyer con capacidad para $250 \mathrm{~mL}$. El experimento fue realizado por triplicado y, después de los siete días de incubación en una estufa biological oxigen demand (BOD) con la temperatura del $25^{\circ} \mathrm{C}$, en cada matraz Erlenmeyer, fueron adicionados $100 \mathrm{~mL}$ de solución Tween 20 a una concentración de $0,05 \%$. Los matraces fueron agitados vigorosamente y fueron tomadas nueve alícuotas de $10 \mathrm{~mL}$ de cada uno Erlenmeyer para la cuantificación de esporas bajo microscopia de luz con aumentos de 10 y 40 veces, con la ayuda de una cámara de Fuchs Rosenthal. El promedio de esporas contenidas en las nueve alícuotas fue considerado para estimar la concentración promedio de las esporas producidas. 


\section{RESULTADOS}

Los aislados fúngicos AC001 y CG722 presentaron las mejores producciones de estructuras reproductivas en el medio QA $(p<0,05)$, como observado en la Tabla 1 . El volumen de $20 \mathrm{~mL}$ de masa micelial utilizado como inóculo inicial resultó en una mayor recuperación de las esporas para los mismos aislados (Tabla 1).

El NF34A presentó baja o ausencia de la producción de estructuras reproductivas en los diferentes volúmenes de masa micelial utilizados (Tabla 1).

El aislado CG722 mostró una producción más amplia de conidios y clamidosporas cuando $20 \mathrm{~mL}$ de la masa micelial fueron agregados en 100 gramos de sémola de arroz $(\mathrm{p}<0,05)$; mientras que el aislado AC001 obtuvo una producción intermediaria, la cual no mostró diferencia estadística con el NF34A ( $p>0,05)$, como se nota en la Tabla 2.

Dentro de los medios sólidos de cultivo evaluados (QA, QM, BC, PA, CC), el QA presentó la mayor producción de estructuras fúngicas y el $\mathrm{BC}$ la menor de conidios y clamidosporas (Tabla 1).
Cuando comparamos los diferentes volúmenes de micelio fúngico utilizados como inóculos (10,15 o $20 \mathrm{~mL}$ ), la mayor producción de conidios y clamidosporas fue observada al inocular $20 \mathrm{~mL}$ de masa micelial sobre los medios sólidos de cultivo (Tablas 1 y 2 ).

\section{DISCUSIÓN}

ARAújo et al. (2004) mencionaron que los hongos depredadores no son exigentes en su nutrición en cultivo y no necesitan de medios complejos. Sin embargo, la producción de conidios y/o clamidosporas por estos organismos es un factor importante, una vez que es necesaria una gran cantidad de esporas para producir formulaciones empleadas en programas de control biológico, a la propagación y supervivencia de los hongos en condiciones ambientales (ARAÚJO et al., 2001). En el presente estudio, se observó que la producción de estructuras fúngicas se logró por medio del uso de medios alternativos, de la

Tabla 1. Promedio de esporas producidas por diferentes aislados fúngicos en variados medios sólidos de cultivo después de siete días de incubación a $25^{\circ} \mathrm{C}$.

\begin{tabular}{|c|c|c|c|c|}
\hline \multirow{2}{*}{$\begin{array}{l}\text { Inóculo } \\
(\mathrm{mL})\end{array}$} & \multirow{2}{*}{$\begin{array}{l}\text { Medio de } \\
\text { cultivo }\end{array}$} & \multicolumn{3}{|c|}{ Aislados fúngicos } \\
\hline & & $\mathrm{ACOO} 1$ * & NF34A* & CG722* \\
\hline \multirow{5}{*}{10} & QA & & $1,1 \times 10^{4}\left( \pm 3,3 \times 10^{4}\right)^{a}$ & $2 \times 10^{5}\left( \pm 4 \times 10^{5}\right)^{a}$ \\
\hline & QM & $7,3 \times 10^{4}\left( \pm 2 \times 10^{5}\right)^{a}$ & $\mathrm{O}^{\mathrm{ab}}$ & $1,1 \times 10^{3}\left( \pm 3,3 \times 10^{3}\right)^{a}$ \\
\hline & $\mathrm{BC}$ & $2,2 \times 10^{3}\left( \pm 4,1 \times 10^{3}\right)^{a}$ & $1,1 \times 10^{4}\left( \pm 3,3 \times 10^{4}\right)^{\mathrm{ab}}$ & $\mathrm{O}^{\mathrm{a}}$ \\
\hline & PA & Oa $3,4 \times 10^{4}\left( \pm 1,4 \times 10^{4}\right)^{a}$ & $1 \times 10^{4}\left( \pm 8,6 \times 10^{3}\right)^{a}$ & $\mathrm{O}^{\mathrm{a}}$ \\
\hline & $\mathrm{CC}$ & $2,2 \times 10^{3}\left( \pm 4,1 \times 10^{3}\right)^{a}$ & $7,7 \times 10^{3}\left( \pm 9,7 \times 10^{3}\right)^{a}$ & $2,2 \times 10^{3}\left( \pm 6,6 \times 10^{3}\right)^{a}$ \\
\hline \multirow{5}{*}{15} & QA & $3 \times 10^{5}\left( \pm 6 \times 10^{5}\right)^{a}$ & $\mathrm{O}^{\mathrm{ab}}$ & $5 \times 10^{5}\left( \pm 4 \times 10^{5}\right)^{b}$ \\
\hline & QM & $2,6 \times 10^{4}\left( \pm 2 \times 10^{4}\right)^{a}$ & $\mathrm{O}^{\mathrm{ab}}$ & $\mathrm{O}^{\mathrm{a}}$ \\
\hline & $\mathrm{BC}$ & $\mathrm{O}^{\mathrm{a}}$ & $1,5 \times 10^{4}\left( \pm 1,6 \times 10^{4}\right)^{a}$ & $\mathrm{O}^{\mathrm{a}}$ \\
\hline & PA & $6,3 \times 10^{4}\left( \pm 2,9 \times 10^{4}\right)^{a}$ & $1,1 \times 10^{4}\left( \pm 1 \times 10^{4}\right)^{a}$ & $1,1 \times 10^{3}\left( \pm 3,3 \times 10^{3}\right)^{a}$ \\
\hline & $\mathrm{CC}$ & $5,1 \times 10^{4}\left( \pm 6,7 \times 10^{4}\right)^{a}$ & $6,6 \times 10^{3}\left( \pm 1,3 \times 10^{4}\right)^{a c}$ & $\mathrm{O}^{\mathrm{a}}$ \\
\hline \multirow{5}{*}{20} & QA & $2,5 \times 10^{5}\left( \pm 3 \times 10^{5}\right)^{a}$ & $\mathrm{O}^{\mathrm{ab}}$ & $5,2 \times 10^{5}\left( \pm 6,5 \times 10^{5}\right)^{b}$ \\
\hline & QM & $3,4 \times 10^{4}\left( \pm 2,4 \times 10^{4}\right)^{a}$ & $\mathrm{O}^{\mathrm{ab}}$ & $3,3 \times 10^{4}\left( \pm 6,6 \times 10^{4}\right)^{a}$ \\
\hline & $\mathrm{BC}$ & $\mathrm{O}^{\mathrm{a}}$ & $5,5 \times 10^{3}\left( \pm 8,8 \times 10^{3}\right)^{a}$ & $\mathrm{O}^{\mathrm{a}}$ \\
\hline & PA & $1 \times 10^{5}\left( \pm 7,5 \times 10^{4}\right)^{a}$ & $6,6 \times 10^{3}\left( \pm 7 \times 10^{3}\right)^{a}$ & $\mathrm{O}^{\mathrm{a}}$ \\
\hline & $\mathrm{CC}$ & $1,4 \times 10^{4}\left( \pm 2 \times 10^{4}\right)^{a}$ & $2,4 \times 10^{4}\left( \pm 1,8 \times 10^{4}\right)^{a c}$ & $\mathrm{O}^{\mathrm{a}}$ \\
\hline
\end{tabular}

Promedios seguidos por diferentes letras minúsculas en las columnas no son estadísticamente diferentes $(p>0,05)$ - prueba de Tukey. QA: sémola de arroz; QM: sémola de maíz; BC: bagazo de caña; PA: paja de arroz; CC: cáscara de café; *valores promedios de recuperación de esporas/ clamidosporas después del período de incubación.

Tabla 2. Promedios de esporas recuperadas después de la incubación de diferentes aislados fúngicos en medios sólidos de cultivo variados después de siete días de incubación.

\begin{tabular}{lccccc} 
Hongos & QA & QM & BC & PA & CC \\
ACOO1 & $2,5 \times 10^{5}\left(3 \times 10^{5}\right)^{\mathrm{a}}$ & $3,4 \times 10^{4}\left(2,4 \times 10^{4}\right)^{\mathrm{a}}$ & $\mathrm{O}^{\mathrm{a}}$ & $1 \times 10^{5}\left(7,5 \times 10^{4}\right)^{\mathrm{a}}$ & $1,4 \times 10^{4}\left(2 \times 10^{4}\right)^{\mathrm{a}}$ \\
\hline NF34A & $\mathrm{O}^{\mathrm{ab}}$ & Oa & $5,5 \times 10^{3}\left(8,8 \times 10^{3}\right)^{\mathrm{a}}$ & $6,6 \times 10^{3}\left(7 \times 10^{3}\right)^{\mathrm{b}}$ & $2,4 \times 10^{4}\left(1,8 \times 10^{4}\right)^{\mathrm{ab}}$ \\
\hline CG722 & $5,2 \times 10^{5}\left(6,5 \times 10^{5}\right)^{\mathrm{ac}}$ & $3,3 \times 10^{4}\left(6,6 \times 10^{4}\right)^{\mathrm{a}}$ & $\mathrm{O}^{\mathrm{a}}$ & $\mathrm{O}^{\mathrm{b}}$ & $0^{\mathrm{ac}}$ \\
\hline
\end{tabular}

Promedios sequidos por letras similares en las columnas no son estadísticamente diferentes ( $p<0,05)$ - prueba de Tukey; QA: sémola de arroz; QM: sémola de maíz; BC: bagazo de caña; PA: paja de arroz; CC: cáscara de café; el inóculo inicial para cada hongo fue de 20 mL de una suspensión micelial. 
preparación simple y a un bajo costo de producción, considerados como subproductos de la agroindustria.

Dhingra; Sinclair (1995) afirmaron que un buen medio de cultivo permite gran esporulación y pequeño crecimiento micelial, siendo que, generalmente, la primera es favorecida por el agotamiento nutricional. Esto es una condición adversa del crecimiento vegetativo; sin embargo, los medios pobres en nutrientes con poca fuente de carbono (C) y nitrógeno ( $\mathrm{N}$ ) favorecen la formación de clamidosporas con supresión del crecimiento vegetativo en función del estrés nutricional.

Según Bogus et al. (2005), los mecanismos involucrados en la regulación de la formación de clamidosporas, así como la función exacta de estas esporas, son desconocidos y se demostraron que los medios de cultivo deficientes en nutrientes (MM y CMA) no producen clamidosporas o aquellas inmaduras. Pero, en todos los medios ricos, estimulase la producción de ellas (por ejemplo LB) en cuanto a las esporas producidas en los medios SAB y SAB-HP no se alcanzó la madurez que se obtuvo en aquellas en los medios SAB-GM. En el presente trabajo, los resultados observados se mostraron contradictorios con otras investigaciones. Como ha sido reportado, los medios de cultivo sólidos con mayores niveles de carbono y nitrógeno (QA y QM), que según MAGALHÃES et al. (2006) tienen el 13,95 y 9,11\% de proteína, 0,61 y $0,66 \%$ de alanina, respectivamente, mostraron aquí una gran capacidad de producir estructuras reproductivas, lo que puede significar que otros nutrientes pueden ser limitantes a la producción de conidios y clamidosporas. Asimismo, se observó una elevada carga de estructuras reproductivas en el medio de cultivo QA, probablemente debido a una mayor riqueza de nutrientes presentes en ello, que, según Dijksterhuis et al. (1993), son considerados esenciales al crecimiento y a la esporulación fúngica.

Maciel et al. (2006) evaluaron la producción de D. flagrans (CG768), A. robusta (I31) y M. thaumasium (NF34A) en los medios de cultivo comerciales (agar-agua - AA2\%; corn meal agar - CMA2\%; extracto de levadura - 0,4\%; $\mathrm{K}_{2} \mathrm{HPO}_{4}-0,1 \% ; \mathrm{MgSO}_{4} .7 \mathrm{H}_{2} \mathrm{O}-0,05 \%$; almidón soluble $2 \%$; agar $-2 \%$; YPSSA - $2 \%$ y potato dextrosa agar - $\mathrm{BDA} 2 \%$ ) y observaron que los aislados CG768 y NF34A presentaron el mayor y menor número de esporas en los medios BDA2\% y AA2\%, respectivamente. En lo mismo trabajo, los autores registraron que el aislado I31 tuvo una producción de esporas intermediarias, sin diferencia estadística en relación a los demás ( $>>0,05)$. GARDNER et al. (2000) observaron que la producción de clamidosporas del $D$. flagrans se incrementó en medio Agar Sabouraud dextrosa (8,6 × 105 clamidosporas $\left.\mathrm{mL}^{-1}\right)$ con un $\mathrm{pH}$ entre 5,5 y 7,5 y en un sistema bifásico (medio líquido Mueller-Hinton, 1\%; extracto de levadura,
$1 \% ; \mathrm{K}_{2} \mathrm{HPO}_{4}, 0,3 \% ; \mathrm{C}_{3} \mathrm{H}_{3} \mathrm{O}_{3} \mathrm{Na}, 0,1 \%$; glucosa, $0,05 \%$ MYPG/medio sólido sales de Vogel). Las concentraciones de esporas notadas fueron de 6,2 y $5,6 \times 10^{5} \mathrm{~mL}^{-1}$, respectivamente, con la adición de almidón o glicerol. En relación al presente trabajo, se observó una similitud en los resultados obtenidos, donde los medios de cultivo pobres (subproductos fibrosos de la agroindustria) propiciaron una baja producción y los ricos en nutrientes esenciales (subproductos energéticos/proteicos) fueron más eficientes en la producción de estructuras reproductivas.

Según Mota et al. (2003), después de la preservación con diferentes métodos de conservación, $A$. robusta (I31) presentó una producción más amplia de estructuras que $M$. thaumasium (NF34A) en medio de cultivo YPSSA, aunque otra gran ha sido observada en ambos aislados. Sin embargo, esos resultados no concuerdan con los registrados en el presente trabajo, una vez que el NF34A presentó una pequeña o nula producción de esporas en medios pobres en aminoácidos y ácidos grasos que, para Dijksterhuis et al. (1993), es considerado esencial al crecimiento y a la esporulación. Según Magalhấes et al. (2006), el bagazo de caña, la paja de arroz y la cáscara de café presentan 1,82, 5,00 y 10,07\% de proteína y 3,99, 12,15 y 17,93\% de ácido graso oleico, respectivamente, y son considerados como forrajes. Aunque Scholler; Rubner (1994) han definido que la formación de clamidosporas es un síntoma de la condición de crecimiento desfavorable, eso efecto no fue observado en el presente trabajo.

\section{CONCLUSIÓN}

La producción de esporas mediante la utilización de subproductos agroindustriales se mostró eficiente con cierta viabilidad técnica y económica. Aquellos con mayores densidades proteica y energética, por ejemplo, la sémola de arroz, promovieron una eficiencia más amplia para la producción de estructuras reproductivas, así como el mayor inóculo inicial.

\section{AGRADECIMIENTOS}

Los autores agradecen a la Fundação de Amparo à Pesquisa do estado de Minas Gerais (FAPEMIG), la Coordenação de Aperfeiçoamento de Pessoal de Nivel Superior (CAPES) y al Conselho Nacional de Desenvolvimento Cientifico e Tecnológico (CNPq) por el apoyo financiero para la ejecución de este trabajo. 

REFERENCIAS

ARAÚJO, J.V.; CAMPOS, A.K.; PAIVA, F.; VIEIRA-BRESSAN, M.C.R. Efeito antagonista de fungos predadores do gênero Arthrobotrys sobre larvas infectantes de Oesophagostomum radiatum, Cooperia punctata e Haemonchus placei. Revista Brasileira de Ciência Veterinária, v.8, p.81-84, 2001.

ARAÚJO, J.V.; MOTA, M.A.; CAMPOS, A.K. Controle biológico de helmintos parasitos de animais por fungos nematófagos. Revista Brasileira de Parasitologia Veterinária, v.13, p.165$169,2004$.

BOGUS, M.I.; CZYGIER, M.; KEDRA, E.; SAMBORSKI, J. In vitro assessment of the influence of nutrition and temperature on growing rates of five Duddingtonia flagrans isolates, their insecticidal properties and ability to impair Heligmosomoides polygyrus motility. Experimental Parasitology, v.109, p.115$123,2005$.

BRAGA, F.R. Ação in vitro de fungos das espécies Duddingtonia falgrans, Monacrosporium sinense e Pochonia chlamydosporia sobre ovos de Fasciola hepatica e Schistosoma mansoni. 2008. $67 \mathrm{f}$. Dissertação (Mestrado em Medicina Veterinária) - Universidade Federal de Viçosa, Viçosa, 2008.

CRUZ, D.G.; ARAÚJO, F.B.; MOLENTO, M.B.; DAMATTA, R.A.; DE PAULA SANTOS, C. Kinetics of capture and infection of infective larvae of trichostrongylides and free-living nematodes Panagrellus sp. by Duddingtonia flagrans. Parasitology Research, v.109, p.1085-1091, 2011.

DHINGRA, O.D.; SINCLAIR, J.B. Basic plant pathology methods. 2 ed. Boca Raton: CRC Press, 1995.

DIJKSTERHUIS, J.; HARDER, W.; VEENHUIS, M. Proliferation and function of the microbodies in the nematophagous fungus Arthrobotrys oligospora during growth on oleic acid or D-alanine as the sole carbon source. FEMS Microbiology Letters, v.94, p. 1 9, 1993.

GARDNER, K.; WIEBE, M.G.; GILLESPIE, A.T.; TRINCI, A.P.J. Production of chlamydospores of the nematode-trapping Duddingtonia flagrans in shake flask culture. Mycological Research, v. 104, p.205-209, 2000.

LARSEN, M. Biological control in a global perspective - a review with emphasis on Duddingtonia flagrans. In: LARSEN, M. Biological control of nematode parasites of small ruminants in Asia. Roma: FAO, 2002. p.19-37.

LARSEN, M. Studies on the capacity of microfungi to destroy animal parasitic nematode. 1991.61f. Thesis (PhD) - The Royal Veterinary and Agricultural University, Copenhagen, 1991.
LIU, X.Z.; ZHANG, K.Q. Nematode-trapping species of Monacrosporium with special reference to two new species. Mycological Research, v.98, p.862-868, 1994.

MACIEL, A.S.; ARAÚJO, J.V.; CAMPOS, A.K. Viabilidade sobre larvas infectantes de Ancylostoma spp dos fungos nematófagos Arthrobotrys robusta, Duddingtonia flagrans e Monacrosporium thaumasium após esporulação em diferentes meios de cultura. Revista Brasileira de Parasitologia Veterinária, v.15, p. 182-187, 2006.

MAGALHÃES, K.A.; VALADARES FILHO, S.C.; PEREIRA, O.G. Tabelas de composição de alimentos. In: Exigências nutricionais de zebuínos e tabelas de composição de alimentos BR-corte. Viçosa: UFV, 2006. p.95-142.

MOTA, M.A.; CAMPOS, A.K.; ARAÚJO, J.V. Influence of different storage methods on the predatory capacity of the fungi Arthrobotrys robusta and Monacrosporium thaumasium after passage through the bovine gastrointestinal tract. World Journal of Microbiology and Biotechnology, v. 19, p.913-916, 2003.

SANYAL, P.K.; SARKAR, A.K.; PATEL, N.K.; MANDAL, S.C.; PAL, S. Formulation of a strategy for the application of Duddingtonia flagrans to control caprine parasitic gastroenteritis. Journal of Helminthology, v. 82, p. 169-174, 2008.

SAXENA, G.; MITTAL, N. Trap formation by conidia of nematodetrapping Monacrosporium spp. Mycological Research, v.7, p.839-840, 1995.

SCHOLLER, M.; RUBNER, A. Predacious activity of the nematode destroying fungus Arthrobotrys oligospora in dependence of the medium composition. Microbiological Research, v.149, p.145-149, 1994.

SILVA, M.E.; ARAÚJO, J.V.; BRAGA, F.R.; SOARES, F.E.F.; RODRIGUES, D.S. Control of infective larvae of gastrointestinal nematodes in heifers using different isolates of nematophagous fungi. Revista Brasileira de Parasitologia Veterinária, v.22, p.78-83, 2013.

TAVELA, A.O.; ARAÚJO, J.V.; BRAGA, F.R.; SILVA, A.R.; CARVALHO, R.O.; ARAÚJO, J.M.; FERREIRA, S.R.; CARVALHO, G.R. Biological control of cyathostomin (Nematoda: Cyathostominae) with nematophagous fungus Monacrosporium thaumasium in tropical southeastern Brazil. Veterinary Parasitology, v.175, p.92-96, 2011.

WALLER, P.J.; FAEDO, M.; ELLIS, K. The potential of nematophagous fungi to control the free living stages of nematodes parasites of sheep: towards the development of a fungal controlled release device. Veterinary Parasitology, v. 102, p.321-330, 2001.

ZHANG, K.; LIU, X.; CAO, L.; GAO, R. A new species of Arthrobotrys from China. Mycological Research, v.100, p.527-530, 1996. 